\title{
VALORACIÓN DE LA TRADICIÓN ORAL COMO FUENTE EN EL ESTUDIO DE NUBES Y TIPOS DE TIEMPO
}

\author{
Alfredo Ramón Morte
}

\begin{abstract}
RESUMEN
La tradición oral puede aportar informaciones complementarias e incluso decisivas en el estudio del clima; además, su cualidad de sintetizar la percepción empírica durante siglos de experiencias humanas en el medio natural, incrementa su valor para la Geografía. En este trabajo se pretende analizar las apreciaciones populares sobre las formaciones nubosas y su relación con determinados tipos de tiempo.

RÉSUMÉ

La tradition orale peut nous offrir des renseignements complémentaires, parfois décisifs, pour etudier le climat. En plus, celle-ci a la particularité de synthétiser la perception empirique, pendant des siècles, des expériences humaines dans le cadre de la nature et ces connaissances sont très importants pour la Géographie. Ce travail a pour but faire une analyse des appréciations populaìres sur les formations nébuleuses et leur rapport avec certains types de temps.
\end{abstract}

El criterio para la elaboración de clasificaciones o atlas de nubes responde a fundamentos principalmente morfológicos, clases y especies que se derivan de diez géneros $^{1}$ elementales cuya identificación obedece a las siguientes características: forma, dimensión, estructura, textura, iluminación y color. «Una clasificación de las nubes debería ser genética, es decir basada en la manera en que se originan las nubes. No se les conocen por desgracia aún suficientemente como para poder establecer una tal clasificación que correría el riesgo, por otro lado, de quedar rápidamente superada $»^{2}$. Por esta razón, la pretensión de este artículo se limita a valorar una fuente más en el estudio de la humedad atmosférica, la tradición oral. Las formaciones nubosas ocupan un lugar destacado entre los mecanismos atmosféricos percibidos y vividos por el hombre, como fieles indicadores de determinados

${ }^{1}$ Cirrus, cirrocumulus, cirrostratus, altocumulus, altostratus, nimbostratus, stratocumulus, stratus, cumulus y cumulonimbus.

${ }^{2}$ CAPEL MOLINA, J. J.: «Nubes cumuliformes», Paralelo 37, n. 4, Almería 1980. 


\begin{tabular}{|c|c|c|c|c|}
\hline \multicolumn{5}{|c|}{ Cawne! } \\
\hline costian & Bencis: & Fanlivis & Neass stes & TKES MNEAS \\
\hline Ćm & 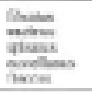 & mones & mans & 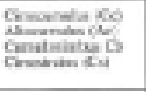 \\
\hline Ansian:ensa & 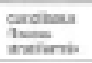 & maina & and & 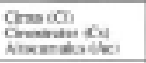 \\
\hline Oremancer & Tos: & going: & & 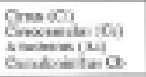 \\
\hline athoum $=|\mathrm{s}|$ & 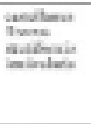 & 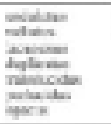 & nons & 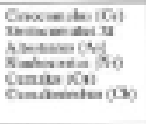 \\
\hline Absertsos iso & & 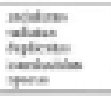 & mins & 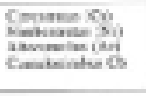 \\
\hline 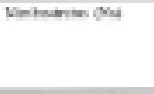 & & & mow & 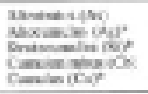 \\
\hline 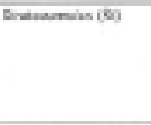 & intion & 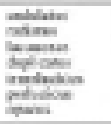 & iment & 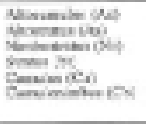 \\
\hline $\operatorname{san}(\infty)$ & Ehtest & mencisis & manciese & 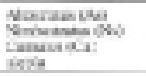 \\
\hline Conds $x=1$ & 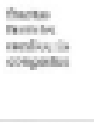 & netime & 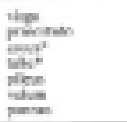 & 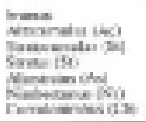 \\
\hline Canavemisicos & ape & & 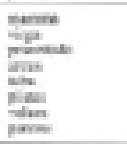 & 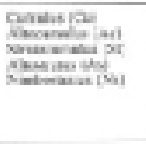 \\
\hline
\end{tabular}

\section{* Sucede en raras ocasiones}


tipos de tiempo de gran trascendencia para una sociedad rural o marinera. Esta circunstancia queda muy bien reflejada en el refranero de la provincia de Alicante ${ }^{3}$ como una forma oral muy depurada de transmisión de saber, cultura, tradiciones y supersticiones, y bien analizada, pese a la fuerte carga subjetiva que hay en ella, puede resultar extraordinaria por su información.

Este ha sido el objetivo perseguido al estudiar una selección de los refranes que hacían referencia a nubosidad y humedad atmosférica entre un total de 1.700 dichos sobre el clima de la provincia de Alicante, resultado de las encuestas realizadas en alrededor de 70 términos municipales. Gracias a ello y con las descripciones contenidas en los apotegmas, se han identificado numerosas y peculiares denominaciones referidas a diferentes géneros, especies y variedades de nubes, pero lo que es más importante, en muchos casos se establece una concordancia directa entre éstas y el tipo de tiempo que preludia su aparición en el cielo, de manera que el factor genético puede intuirse con bastante frecuencia. Para facilitar la lectura de este trabajo se incluye una detallada descripción de los diferentes géneros, especies y variedades de nubes (ver cuadro I).

\section{Borrascas frontales y procesos de inestabilidad sobre el Mediterráneo}

Los cirrus son conocidos en la cuenca del río Amadorio como «camins» (caminos), es decir, radiatus, o también como «rams» (ramos), cirrus fibratus, bien sean intortus o vertebratus; en el Bajo Vinalopó también se conocen como «gambirots» (brincos). Este género pertenece a las nubes de mayor altura, de aspecto fibroso y brillo sedoso, con inconfundibles formas filamentosas muy variadas y caprichosas, constituidas por cristales de hielo que ofrecen la particularidad de refractar la luz oblicua del Sol, de esta manera, en el orto o en el ocaso adoptan una coloración amarillenta o intensifican las tonalidades rojizas del cielo y el aerosol atmosférico, de ahí la denominación de «arrebols», circunstancia muy frecuente ante la llegada de la cabeza del sistema nuboso asociado al paso de un anafrente cálido, con un aumento progresivo de la inestabilidad atmosférica en forma de viento e incluso precipitación, fenómenos aludidos en los adagios siguientes: «Arrebols a tot cap, oratge endiablar» (Arreboles por todos lados, tiempo endiablado), «Camí rosat, als tres dies banyat» (Camino rojizo, a los tres días bañado), «Posta roja o sol rogenc, Ponent al dia següent» (Puesta roja o sol rojizo, viento Poniente al día siguiente).

Sin embargo, a diferencia de lo que seguramente ofrecería la tradición oral de regiones atlánticas, en esta zona del Mediterráneo, a sotavento de la circulación dominante del oeste propia de la zona templada, los frentes provocados por estas células ciclónicas suelen deparar escasas repercusiones pluviométricas, por un claro proceso de frontólisis. Debido a ello los «arrebols» no siempre se identifican con la inminencia de lluvia, sino más bien con circulaciones superficiales: «Por la tarde arreboles, por la mañana soles», «Arrebols de nit, sol pel coati» (Arreboles de noche, viento por la mañana), «Sol i núvols roigs al Ponent, senyal de vent» (Sol y nubes rojas al Poniente, señal de viento), «Nube amarillenta, viento representa», "Reboleras de tarde, viento de mañana», "Si la posta del sol es encarnà (rogenca), s'espera una bona aventà (aventada)» (Si la puesta del sol es rojiza, se espera una buena ventisca); en la isla de Tabarca a los cirrus de la especie uncinus, en forma de ganchos, conocidos como «asmolaores» ${ }^{4}$ se les atribuye la posibilidad de barruntar el soplo del Mistral (NW) en la llegada de un frente frío, sin embargo los «rabeis de gall»o

\footnotetext{
${ }^{3}$ Durante un período de dos años se ha llevado a cabo una intensa labor de recopilación del refranero climático, todavía vivo en muchas poblaciones de la provincia de Alicante.

${ }^{4}$ No tiene traducción.
} 
cirrus de la variedad vertebratus se presumen como indicadores del soplo del «Llebeig» (Lebeche SW), viento portador de una masa de aire menos fría. Esta relación de los cirrus arrebolados con circulaciones eólicas se advierte en otro calificativo aplicado a las nubes irisadas: «bandera flamenca».

Estos últimos ejemplos contrastan con los que se refieren a las tonalidades rojizas de los cirrus iluminados oblicuamente desde el este: «Aurora o sol rogenc, pluja o vent» (Aurora o sol rojo, lluvia o viento), "Arrebols pel matí, a la nit aigua ací» (Arreboles por la mañana, a la noche agua aquí), "Cel rogenc per la vesprá, la pluja se’n aná» (Cielo rojizo cuando ha atardecido, la lluvia ya se ha ido); de hecho, la situación atmosférica es muy diferente, la circulación dominante se ha invertido en superficie y la presencia de estas nubes anuncia con cierta antelación la evolución de un frente de inestabilidad generado sobre el Mediterráneo, con aire húmedo y de mayor efectividad pluviométrica, motivo del protagonismo de los hidrometeoros sobre los eolometeoros. "El Ponent la mou i el Llevant la Plou» (El Poniente la mueve y el Levante la llueve), "Las nubes que en una hora saca el Levante, en una hora las mete el Poniente», de ahí que en el Bajo Segura al tiempo seco y frío se le denomine «tiempo de Poniente» y al lluvioso «tiempo de Levante».

Por supuesto que la observación de cirrus se puede relacionar con tipos de tiempo muy variados, pero en una región de considerables condiciones de aridez y en un ejercicio prolongado durante siglos, sus habitantes procuran dilucidar y reconocer aquellas situaciones que son el preludio de precipitación. Además, pueden servir para completar la información sobre las condiciones de un tipo de tiempo, o incluso orientar la elaboración de hipótesis a la hora de investigar la naturaleza de la masa de aire en determinadas situaciones sinópticas.

Los cirrostratus son un velo nuboso blanquecino, extenso y continuo, que puede presentar aspecto fibroso (fibratus) o liso (nebulosus), pero siempre compuesto por una fina y homogénea capa de cristales de hielo con la suficiente transparencia como para permitir que durante el día la luz del sol pueda proyectar la sombra de los objetos, sin embargo, en el horizonte adoptan una coloración grisácea y se pueden confundir fácilmente con altostratus. La presencia en lontananza de nubes alargadas de color rojo y pardo, llamadas en el Bajo Segura «bardas», o «bigotis», «closca de ceba»y «roca» en el Bajo Vinalopó, con tonalidades que las confunden con el relieve del horizonte, hace pensar en cirrus spisatus duplicatus, cirrostratus de las especies fibrosus o nebulosus y de las variedades undulatus y duplicatus, o en altostratus, siempre como evidencia de un alto grado de inestabilidad de la atmósfera asociado igualmente al paso de una borrasca frontal: "Cuando el sol tiene bardas el jueves, llueve antes del domingo», "El divendres sol poniente $i$ en roca, el dilluns aigua fins la bajoca» (el viernes sol poniente y en roca, el lunes agua hasta la bajoca -comparación con el pene-) «Sol entre nubes rojas y pardas sol de aguas». Otra denominación aplicada a esta situación en el Bajo Vinalopó y que resulta muy evocadora por su relación con lluvias es: «banyes» (baños). Desmesurada preocupación por prever la precipitación que va más allá del problema de la aridez y las producciones agrarias, puesto que se trata de comarcas situadas en cuencas bajas con zonas inundables y de unos cauces de funcionamiento irregular proclives al desbordamiento.

La característica fundamental de los cirrostatus, dada su composición, es la refracción y reflexión de la luz al atravesar los prismas exagonales de hielo, los rayos incidentes se concentran alrededor del foco luminoso lunar o solar para formar el fotometeoro llamado halo, anillo rojo que hacia sus bordes adopta una tonalidad violácea, fenómeno que en el Campo de Alicante se conoce como «gandufa», en la montaña alicantina como «rogle», «rotgle» o «rogle»; en el Bajo Segura como «círculo», «rolde» o «cerco»; en el Campo de Elche como «rotillo» o «rotlle» y en el Alto Vinalopó como «gayufa», nombres asociados a un cambio de tiempo con incremento de la inestabilidad: "Círculo de sol, moja al pastor», 
«Lluna rotllada, el temps mudará» (Halo de luna, cambio de tiempo), «Lluna roldada (enrondada), als tres dies banyada» (Luna con halo, a los tres días bañada). En ciertas ocasiones, la orientación de los cristales de hielo dentro del cirrostratus aparece desordenada debido a fuertes corrientes de aire y turbulencias en los altos niveles de la troposfera, ello favorece la formación de parhelios o paraselenes, según se trate del sol o de la luna, conocidos en la costa como «ressol» y que en realidad son focos secundarios de luz brillante alrededor del círculo parhélico semejantes a «estrellas» dentro del halo: "Luna roldá con estrellas en su centro, si a los tres días no llueve, Levante cierto», "En llevar la luna cerco con estrellas dentro, agua o viento», "Lluna amb estrella, guardat d' ella» (Luna con estrella, guárdate de ella); es lógico pensar que las fuertes circulaciones de altura pronto puedan dejar sentir sus efectos en superficie.

La formación de cirrostratus se produce en el ascenso lento de una capa de aire de gran extensión horizontal que de existir condiciones atmosféricas favorables puede dar lugar a altostratus, altocumulus y nimbostratus, o generarse en la parte alta de un cumulonimbus, en cuyo caso la precipitación puede ser más intensa e inminente. No obstante, sólo representan una posibilidad de evolución hacia un tipo de tiempo inestable, pero en este ámbito geográfico siempre ejerce gran influencia la preocupación subjetiva por la posibilidad de precipitación.

\section{Convección de la masa de aire forzada por el relieve}

Al igual que los dos géneros anteriores, los cirrocumulus son nubes propias de los niveles más altos de la troposfera, compuestas por cristales de hielo y minúsculas gotas de agua en subfusión, cuya transparencia permite contemplar los astros y en determinadas ocasiones la formación de fenómenos de corona. Sin embargo, a nivel popular se relacionan con el género altocumulus por guardar cierta similitud, pese al mayor tamaño de éste último y la cualidad de producir sombras propias. Las especies floccus y lenticularis suelen tener origen en la convección forzada por el relieve sobre una capa de aire húmedo, lo que en ésta parte del Mediterráneo manifiesta un claro proceso de inestabilidad, en especial durante las épocas equinocciales. Ubicadas en el piso medio, entre 2 y $6 \mathrm{Km}$ de altura y formadas por gotillas de agua, la especie altocumulus floccus puede producir virgas de cristales de hielo y por lo tanto fenómenos de halo, pero lo verdaderamente característico es la posibilidad de provocar la difracción de la luz del sol o la luna, formando en torno a ellos una gran corona luminosa (con gradación de colores inversa a la del halo). A medida que la inestabilidad y la condensación de las gotas dentro de la nube aumente, la corona se reducirá de tamaño, método popular para determinar la posibilidad de lluvia y la evolución de la condensación dentro de la nube: «Lluna amb corona, aigua dona» (Luna con corona, agua da).

Resulta muy conocida la peculiar forma de este género en su estado más puro, compuesta por formaciones de individuos similares, de aspecto algodonoso y dispuestos de una manera ordenada, como un muro de ladrillos («cielo empedrado») o un rebaño de corderos, de ahí la denominación «cel de cotó» (cielo de algodón) o «cel a borreguets» (cielo de corderitos): «Cel de cotó, Llevant bufader»(Cielo..., Levante soplador), «Cielo enladrillado veinticuatro horas mojado», "Cel a borreguets, aigua a cantarets» (cielo con corderitos, agua a cantaritos), «Cel a borreguets, neu o aigua a cabaçets» (cielo..., nieve o agua a capacitos), "Cielo borreguero, vendaval o agua del cielo», "Cel emborregat, als tres dies banyat» (cielo aborregado, a los tres días mojado). La variedad lacunosus de los géneros 
altocumulus o cirrocumulus, permite contemplar entre sus intersticios pequeñas porciones de cielo llamadas popularmente «clariana», y cuando dejan pasar los rayos del sol se conocen como «ulls de boc» (ojos de cabrón), si los haces luminosos son de luna, el nombre cambia a «ulls de perdigot» (ojos de perdigón). La aparición del género altocumulus suele coincidir con el flanco de un sistema nuboso depresionario o con turbulencias en la media troposfera, en las que el relieve juega un importante papel, así, vientos de Xaloc (SE) y de Llevant (E) transportando una masa con una fuerte carga de humedad relativa y potencialmente inestable, suelen ser responsables de la condensación en el piso medio.

Los altocumulus de la especie lenticularis y los stratocumulus o cumulus anclados por barreras orográficas del litoral, constituyen la mejor evidencia del ascenso forzado de una masa de aire húmedo e inestable, no en vano en la práctica totalidad de la provincia, existe gran número de refranes referidos a la llegada de un temporal de lluvias intensas cuando estas nubes coronan una u otra sierra, siempre utilizando peculiares denominaciones como «barret» (sombrero), «blanura» (blancura), «capa», «capell», «capella», «capot» (capote), «capolet», «cassola» (cazuela), « follerat -forrellat-« (cerrojo), «pontet» (puentecito), «mantell», «mantellina» y «martell»: «Núvols en Bernia, Llevant segur» (Nubes en la sierra de Bernia, viento de Levante seguro), «Boira pels tossals, aigua pels barrancs» (Neblina en los altos, agua por los barrancos), "Cabeçó coronat, dins de tres dies banyat» (Sierra del Cabeçó d'Or coronada, dentro de tres días bañada), «Cabeçó, Maigmó y Ventós tapats, demá plourá», (Sierras del Cabeçó, Maigmó y Ventós tapadas, lloverá mañana), «Forrellat tancat damunt del Cabeçó d'Or, pluja» (Cerrojo echado en lo alto del Cabeçó, lluvia), «Boireta al Maigmó, aigua al faldó» (Nieblecilla en el Maigmó, agua en el faldón), «Boira en la Penya (Penyaroja), aigua a l'esquena» (Niebla en la Peñarroja, agua en la espalda), "Cuando San Pascual -pico- se pone el gorro y el Cid-sierra-la montera, llueve aunque Dios no quiera», "Cuando Camara-sierrase enoja, Elda se moja», «Boireta en la Serra d'Orxeta, aigua segureta» (Neblina en la Sierra de Orxeta, agua aseguradita), "Quan Ifac -peñón- porta gorreta, aigua segureta» (Cuando Ifac lleva gorrita, agua segurita), "Quan Serrella fa capella, beu-te l'aigua i guardat d'ella»(Cuando la Sierra de Serrella se encapota, bébete el agua y guárdate de ella), "Quan Segària porta capa i el Montgó capot, vés cap a casa llaurador, que no et faltará raó» (Cuando Segaria lleva capa y el Montgó capote, vete a casa labrador que no te faltará razón), "Quan Montgó es posa la capa i Bernia la mantellina, ningú al temporal escapa ni te ronda la fadrina» (Cuando Montgó se pone la capa y Bernia el capotillo, ninguno al temporal escapa, ni se sale a rondar a la novia), «Si al Puigcampana el núvol es posa, tronada ens toca» (Si se pone una nube en el Puigcampana, tormenta nos toca).

Merece la pena destacar la costumbre de hacer pleita de esparto durante los días de «mal tiempo», para rentabilizar las jornadas perdidas en el trabajo agrario, así: «Si Mariola fa cassola y Cestulles capell, pica espart i fes cordell» (Si Mariola tiene una cacerola y Cestulles un gorro, pica esparto y haz cordel), "Quan Puigcampana té capell, pica espart i fes cordell» (Cuando el Puigcampana tiene sombrero, pica esparto...). De esta manera, se aplican formulas parecidas en el resto de los refranes hasta referenciar las nubes orográficas más comunes en toda la provincia de Alicante, aspecto de enorme valor geográfico puesto que constituye una detallada descripción de como el relieve de las distintas comarcas interviene en los procesos de inestabilidad atmosférica.

Los géneros estudiados hasta este momento se utilizan popularmente para averiguar el grado de inestabilidad de la atmósfera a corto plazo, resultando las precipitaciones de la evolución hacia otras formaciones nubosas más representativas de un tipo de tiempo inestable. En los próximos casos las nubes a estudiar son las auténticas protagonistas de la precipitación en sus diferentes formas y, a menudo, la verificación de los vaticinios establecidos 
en los refranes anteriores. Así, el género altostratus sirve para denominar a las nubes grises o azuladas, de aspecto fibroso, estriado y uniforme, que cubren grandes extensiones de la bóveda celeste y que están formadas por cristales de hielo, gotillas de agua, de lluvia y por copos de nieve; que por la existencia de capas de aire turbulento y húmedo pueden presentarse con nubes anejas del tipo pannus (conocidos popularmente como «núvols penjollosos»), es decir, colgajos o jirones desgarrados que se pueden unir conforme el altostratus aumenta de tamaño para formar una capa superpuesta a la base, variedad duplicatus, lo que muestra un claro empeoramiento del tiempo: «Núvols sobrepots y penjollosos, aiguasons (xàfecs) copiosos» (Nubes superpuestas y con colgajos, aguaceros copiosos).

La composición de los altostratus no permite normalmente la formación de halos o coronas, pero cuando no es excepcionalmente denso, como ocurre con la variedad translucidus, permite la visión deslustrada de la luna y los astros como si se tratase de un cristal esmerilado, efecto luminoso que se conoce como «truita» y en algunas zonas de la Marina Baja como «velada de sol»: «Estrellas tristes o veladas, lluvias o granizadas», "Luna descolorida, aire y mar embravecida», «Estrèles opaques i lluna (somorta) mortecina, la plúja s'aveïna» (Estrellas opacas y luna mortecina, la lluvia se avecina), «Lluna morta, Llevant (o aigua) a la porta» (Luna muerta, Levante (o agua) a la puerta), «Luna pálida, anuncia agua, luna roja viento y blanca, buen tiempo», «Sol i núvols formen un vèl, que no deixa vore el cel» (Sol y nubes forman un velo, que no deja ver el cielo).

Los altostratus translucidus que permiten la contemplación deslustrada de la luz que los atraviesa, pueden surgir por la circulación de vientos marítimos de componente este, advecciones que al llegar a la costa llegan a evolucionar hasta incluso cumulus y cumulonimbus ligados a procesos tormentosos con granizo, a los que acompaña un sonido peculiar, el «remor», que se produce por el choque de los granos de hielo dentro de la nube. Cuando desde el mar se cierne sobre la costa un cumulonimbus que tiene estratificada alguna parte de la nube en forma de altostratus y que presenta nubes suplementarias del tipo pileus o arcus, los habitantes de la zona afectada saben que la tormenta puede ser muy violenta, por ejemplo, en las zonas interiores del Bajo Segura a un cumulonimbus con zonas estratificadas se le denomina «milhoja», por su similitud con un pastel dispuesto en capas de hojaldre y crema, e indica la posibilidad de un proceso tormentoso: "Milhojas por Alicante, tormenta enfadá». En el caso de que las condiciones de la alta troposfera no sean favorables al ascenso convectivo de la masa de aire, lejos de precipitar pueden formar cirrostratus o simplemente altocumulus indicativos de una relativa elevación local del flujo marítimo de Levante; por ello las alusiones a la lluvia son menos contundentes que en el caso de las variedades duplicatus y opacus, no obstante siempre subyace el protagonismo del Mediterráneo en la formación de tiempos inestables con precipitación.

El altostratus se produce por la ascensión lenta de una extensa capa de aire, pero en condiciones muy frías de la media troposfera también puede evolucionar a partir de altocumulus soldados que presentan nubes anejas formadas por regueros de cristales de hielo tipo virga; por ello, en los momentos culminantes de una ola de frío y ante la llegada de una masa de aire polar o ártica que pueda encontrar cierto grado de humedad, con viento Mistral (NW) o una Tramuntana-gregalada (N-NW) en superficie, este género y sus estadios intermedios hacia el nimbostratus anuncian una inminente precipitación sólida en forma de nieve en las zonas montañosas del interior de la provincia. Este es el motivo de que la precipitación nival se asocie al cielo de altostratus y nimbostratus, conocido como «Cel de panxa de burra» (cielo de panza de burro) por las tonalidades grisáceas que en el caso de los primeros son más claras y muy similares con el pelaje de esta parte del animal: «Cel de panxa de burra, pluja segura»(Cielo..., lluvia segura), «Cuando va a nevar, el cielo tiene panza de burro». 


\section{Nubes bajas y procesos de inestabilidad}

Si la lluvia o la nieve llegan al suelo se trata generalmente de nimbostratus, género que guarda íntima relación con el altostratus y que alude a nubes compuestas por partículas sólidas y líquidas, gotas de agua y nieve, de un color gris oscuro que cubre todo el cielo y con la particularidad de presentar en su base nubes anejas tipo virga, mamma o praecitado, que ponen de manifiesto su característico enlace con los hidrometeoros. El praecitado que aparece en la base de los nimbostratus, cumulonimbus, cumulus, altostratus y stratus, constituye la mejor evidencia de efectividad pluviométrica, ya que se trata de una auténtica columna de precipitación que alcanza el suelo. Dada la composición de los tres primeros géneros y de las nubes suplementarias que los acompañan, su llegada se observa en la tradición popular por la observación del Arco Iris, fenómeno luminoso provocado con la refracción por dispersión de la luz solar, conocido en las zonas valenciano parlantes como la «rall», «raig», «arc» $\mathrm{O}$ «ratlla de Sant Martí». Con una circulación dominante del oeste propia de la zona templada, la aparición del Arco Iris por la mañana implica una situación occidental de la precipitación y su inminente aproximación; por el contrario, si éste se forma por la tarde la inestabilidad se encuentra a oriente y supone un alejamiento de la misma: «L'arc de Sant Martí pel matí, la plúja ja està ací» (Arco Iris por la mañana, la lluvia ya está aquî), «La ratlla de Sant Martí, pel matí, la plúja és ací; per la vesprada, aigua passada» (El Arco Iris por la mañana, la lluvia está aquí; por la tarde, agua pasada), «Arco al Levante, el agua va delante», «Por la mañana Arco, por la tarde charco», «Arco al Poniente, carga el arado y vente».

El nimbostratus se puede generar por el lento ascenso de una extensa capa de aire, aunque frecuentemente lo haga por el aumento del grosor de una capa de altostratus. Por la parecida apariencia y las abundantes formas de transición entre ambos, muchos de los refranes anteriores pueden estar referidos indistintamente a uno u otro, salvo aquellos que se refieren a imágenes luminosas deslustradas, ya que el nimbostratus no es tan transparente y por lo tanto el color gris es más oscuro. «Nube negra, proa a tierra», «Luna puesta recién salida, a vigilancia convida».

Las nubes del género stratocumulus tienen forma de manto o capa de tono gris blanquecino con sombras propias en forma de baldosas o guijarros de aspecto no fibroso análogo al altocumulus, pero a niveles inferiores. La especie más común es el stratocumulus stratiformis duplicatus en forma de manto continuo, extenso y superpuesto, ya que las especies lenticularis y castellanus se presentan con menor frecuencia y no aparecen en la tradición oral de una manera manifiesta. Sin embargo, un aspecto muy característico es lo que en el Bajo Vinalopó se conoce como nubes que tienen los «Baixos carregats» (bajos cargados), son del tipo mamma, elementos anejos a las de este género y frecuentemente a otros como altocumulus, altostratus y cumulonimbus, denominación alusiva a una característica anatómica femenina que suscita en elBajo Segura los apelativos de «nubes hembras»o «nubes preñadas», de las que se espera lluvia o al menos llovizna.

El stratocumulus es una nube que siempre se origina a partir de la evolución de otras, para ser en muchas ocasiones un estadio de transición, de esta manera si alcanzan cierto grosor (stratiformis opacus) son susceptibles de transformarse en nimbostratus, si no son muy espesas (variedad translucidus) pueden generar fenómenos de corona o convertirse en altostratus; si presentan virgas de cristales de hielo anejas a la base favorecen la formación 
de halos, e incluso podrían evolucionar por la estratificación de algunas partes de altocumulus, cumulus y cumulonimbus; con seguridad esto provoca una difícil identificación y su ausencia de las sentencias populares.

No ocurre lo mismo con las nubes de bajos niveles del género stratus, de tonalidad gris, base uniforme y compuestas generalmente por gotillas de agua, aunque de presentar cierta densidad también integran cristales de hielo y cinarra. Para el refranero, su principal peculiaridad es que cada una de las dos especies en que se manifiestan se relaciona con tipos de tiempo totalmente diferentes. La especie fractus, en forma de bancos desgarrados, al igual que su homóloga del género cumulus, se muestra en jirones con una tonalidad gris oscura y de manera muy similar a una nube de humo por combustión, de gran movilidad y de rápida evolución, pues son formadas a partir de las turbulencias de aire húmedo con precipitación caída desde altostratus, nimbostratus, cumulus y cumulonimbus, y por ello son consecuencia de un tiempo lluvioso y un acertado vaticinio de inmediato «mal tiempo»: «Nube baja y como humo, que lloverá me presumo», «Nubes bajas, anuncian agua». «Boira terrera, aigua sempre espera» (Niebla terrera, agua siempre espera).

Durante las crecientes noches del otoño, cuando el agua del mar por su propio calor específico conserva mayor temperatura que el aire suprayacente o que el aire de la costa, se producen nieblas o neblinas que son el resultado de altas tensiones de vapor sobre el Mediterráneo Occidental, lo mismo ocurre en los valles interiores con vegetación y las zonas húmedas de tierra firme. El fenómeno se incrementa por la advección de masa de aire frío, es decir, la llegada de un frente frío o una vaguada polar o ártica que pueda transformarse en una baja desprendida en altura que utiliza el potencial de energía latente en superficie para generar chubascos de efectos catastróficos en ocasiones, que dejan muy sensibilizados a los habitantes de las comarcas afectadas. A ello aluden los refranes siguientes: "Final de octubre lluvioso, invierno anuncia brumoso», "A las tres relentadas la lluvia no falta», «A las tres escarchas la lluvia no falta», «A las tres borias llueve», "Boires baixes, humetats llargues» (Nieblas bajas, humedades largas), "Quan la boira pastura, aigua segura» (Cuando la niebla pastura, agua segura), «No te fíes de niebla, ni de promesa de suegra». No obstante, en los chubascos producidos por los procesos de «gota fría» el cumulonimbus capillatus se convierte en el auténtico protagonista.

\section{Nubes bajas y estabilidad atmosférica}

La especie nebulosus del género stratus corresponde a la elevación progresiva de una capa de niebla impulsada por un caldeamiento convectivo durante las primeras horas del día, aunque también puede ser provocada por movimientos advectivos superficiales o el arrastre eólico de brumas marinas hacia la costa, fenómeno este último, que se conoce en Tabarca como «pa de boira» (pan de niebla). La saturación del aire se produce con cierta facilidad durante un tiempo anticiclónico de invierno, por enfriamiento nocturno del suelo y de la masa de aire superficial estancada por un cierto abrigo orográfico, sin embargo, con el caldeamiento superficial de la mañana y con la ayuda del soplo de la brisa marina de Levante se eleva y se transforma en stratus, de esta manera el sol al oriente se contempla a través de una capa de gotillas de agua que pueden originar fenómenos de corona solar o dar a la nube una tonalidad blanca brillante, pero sin crear el efecto de un vidrio esmerilado: «Sol blanc, vent al Llevant» (Sol blanco, viento de Levante), "Boira pel matí, sol pel vespre» (Niebla al amanecer, sol al atardecer), «El dia ho escamapa i la nit ho arreplega» (El día lo escampa y la noche lo recoge), "Boires matineres que es disipen, el bon temps pronostiquen» (Nieblas matinales que se disipan, el buen tiempo pronostican), «Boira al matí, 
calma o vent a Garbí» (Niebla de mañana, calma o brisa de Garbino -en algunas zonas costeras de la provincia de Alicante, el Garbino se considera una brisa de componente SE o SSE-).

En estas situaciones de estabilidad, los stratus degeneran en fractostratus y se disipan a lo largo del día, o a lo sumo evolucionan a cumulus humilis o de «buen tiempo» con un cielo azul brillante cuya atonía es sólo interrumpida por estas blancas acumulaciones; así, si el cielo se despeja después de estar nublado, se dice: «Desduir el cel», «Desbarriar-se el temps»o «Estar el cel ras com un ull de peix» (estar el cielo raso como un ojo de pez). Cuando el incremento de la presión no se debe a enfriamientos basales de la capa de aire, sino por la influencia dinámica estival del ascenso latitudinal del Alta de Azores, se hacen frecuentes los fenómenos de calima con ambiente seco. Pero en la costa, la existencia de una masa de aire cálido y húmedo frenada por el techo de inversión genera lo que popularmente se conoce como «basca»: «Dia boirós, humit i calorós» (Día neblinoso, húmedo y caluroso). En estas condiciones, si se llega a producir una tormenta de calor por la rotura de la superficie de inversión a causa de desarrollos cumuliformes durante el día, la precipitación suele ser más bien escasa. «Pluja d'estiu, coixera de gos i plor de bagassa, no et fies massa» (Lluvia de verano, cojera de perro y llanto de fulana, no te fíes mucho) «Pluja d'estiu,... de bagassa, en tant se'n pasa» (Lluvia de verano,... y llanto de fulana, al poco se pasa).

Esta paradoja que presentan stratus y cumulus de aparecer en tipos de tiempo muy diferentes hace que se tienda a afinar en la identificación de sus especies y variedades, por ello, cuando el cumulus es gris y tiene tendencia a desarrollarse en altura (congestus), o cuando el stratus es fractus, el tipo de tiempo es muy diferente al que generan stratus nebulosus o cumulus humilis: «Boina blanca a la vall, homes al treball; boina negra a la colina, homes a la cuina» (Niebla blanca en el valle, hombres al trabajo; niebla negra en la colina, hombres a la cocina). Los conceptos neblina, niebla, nebuloso, nube y nuboso se confunden en la percepción popular, no ocurre lo mismo en Climatología, por esta razón y dada la estrecha relación genética entre nieblas, brumas, stratus y cumulus, en los chascarrillos pueden aparecer bajo la misma denominación de «boira»o «boria».

\section{Nubes de desarrollo vertical y chubascos}

Se han analizado refranes referidos a géneros de nubes desde la alta troposefera hasta las condensaciones superficiales, siempre dentro de un margen altitudinal, pero, a diferencia de éstos los géneros cumulus y cumulonimbus se caracterizan por su desarrollo en la vertical, motivado por corrientes convectivas de origen térmico (tormentas de verano), mecánico (choque contra cadenas montañosas) o dinámico (convergencias en superficie o divergencias en altura). A diferencia de los cumulus humilis, las especies fractus, mediocris y congestus se forman por la existencia de fuerte inestabilidad atmosférica, en especial las dos últimas, conocidas como «torres» 0 «torrejón» en el Alto Vinalopó, temidas por la posibilidad de producir chubascos o de incrementar su violencia hasta formar cumulonimbus. Cuando el cielo está cubriéndose por el desarrollo de un cumulus congestus, nube blanca de gran tamaño y con protuberancias en forma de «coliflor», en el Bajo Segura se dice que el tiempo «está en leche».

El cumulonimbus, al igual que el cumulus, está compuesto por gotillas de agua, aunque en las zonas altas aparecen cristales de hielo, granizo y nieve, coronado por una terminación lisa en forma de yunque (especie calvus) o fibrosa en forma de penacho (capillatus), este último por su desarrollo vertical alargado y su terminación abierta y estriada es 
conocido en el Bajo Segura como «parmera» (palmera) y su contemplación en el horizonte o cercano a alguna sierra litoral es signo del peor augurio: "Parmera en el Carrascoy (sierra costera)...» o «Esta nube es del Carrascoy...» son expresiones típicas de chubascos $\mathrm{e}$ inundaciones. Su desarrollo suele ser a partir de cumulus, de forma aislada o en forma de barrera continua que desde el mar ensombrece la bóveda celeste y sólo permite ver algo de luz entre la superficie del mar y sus bases, entonces los habitantes de la costa aluden a la expresión «la mar s'arrasa» o «gropada»: «Si per la mar s'arrasa, veste'n a casa» (Si por la mar se arrasa, vete a casa), "Quan la mar s'arrasa, ficat dins de casa» (Cuando la mar..., quédate dentro de casa), «Si plou i s'arrasa, no eixiu de casa» (Si llueve y se arrasa, no salir de casa).

Cuando el cumulonimbus capillatus se sitúa encima del observador cubre el cielo casi por completo de un tono oscuro, sombrío y amenazador, de forma parecida a como lo haría un nimbostratus («sebrar-se el cel»o «temps podrit»-tiempo podrido-); el dramatismo ahora se acentúa por la presencia de electrometeoros, situación que en Tabarca se conoce como «grop»: «Nube negra, proa a tierra», "Quan nuvolà fosca corrent se presenta, tenim damunt la turmenta» (Cuando una oscura «nublada» corriendo se presenta, tenemos encima la tormenta)

El cumulonimbus suele aparecer con una gran variedad de nubes anejas o suplementarias, caso de la mamma («nube preñada» o «baixos carregats»), virga («núvols penjollosos»), arcus («milhoja»), pileus, velum, praecitado y tuba, esta última cuando aparece en la base como un cono nuboso invertido puede significar un torbellino ascendente de aire con agua de mar e incluso con pequeños animales, es decir una tromba marina, también conocida en Tabarca como «mar de llamp» (mar de relámpago). Debido a la acumulación de energía calorífica y vapor de agua durante el final del verano y el otoño, una advección de aire frío en altura encuentra las condiciones ideales para disparar los ascensos convectivos sobre el litoral mediterráneo peninsular, con grandiosos desarrollos nubosos verticales hasta el propio techo de inversión que supone la tropopausa, entonces las precipitaciones más abundantes e intensas son la primera consecuencia y a su vez, la causa del peligroso funcionamiento de ramblas y barrancos: «Octubre es mes de historias que deja malas memorias», «Septiembre se tiemble, o seca las fuentes o se lleva los puentes», "Santa Teresa llena las presas». Resulta curioso observar como en los refranes dedicados a las nubes de altura la preocupación por pronosticar una posible precipitación es obsesiva, mientras que en los dedicados a las nubes de desarrollo vertical ésta se da como un hecho y la inquietud se encamina más hacia los efectos que la intensa lluvia trae consigo.

\section{Conclusiones}

Las deducciones que se pueden extraer de este análisis pueden ser muy numerosas, pero la brevedad del artículo impone obviar algunas reflexiones y hacer una jerarquización inevitable. Así, la contemplación de las nubes en un medio de conocida escasez de precipitación obliga a una desmedida preocupación por distinguir aquellas especies o variedades que pueden hacer presumir ciertos hidrometeoros, aunque, pese a la aridez, la irregularidad de las lluvias despierta la necesidad de identificar aquellas nubes que descargan violentos chubascos de extraordinaria trascendencia.

En lo que respecta a la preocupación por los hidrometeoros, la tradición oral no deja lugar a dudas cuando pone de manifiesto la indiscutible importancia del Mediterráneo como elemento principal en la génesis de tiempos inestables de gran eficacia pluviométrica.

Por otro lado, una exhaustiva aparición en los refranes de los géneros cirrus, cirrostratus, 
cirrocumulus, altocumulos y altostratus, responde de manera inconsciente a un análisis de la situación en las capas altas de la troposfera como medio más eficaz de realizar una predicción relativamente anticipada del tiempo.

La percepción popular hace uso de los fotometeoros como si se tratase de una auténtica radiografía de las nubes, por supuesto que desconoce su composición, pero gracias a éstos se permite diferenciar géneros y especies con toda clase de detalles, lo que refleja un grado de conocimiento empírico muy alto. La adscripción de fenómenos atmosféricos a las unidades de relieve, en especial las nubes orográficas, constituye una información geográfica inestimable para los estudios de climas regionales y locales.

En los dichos populares analizados se describen formaciones nubosas presentes en tiempos de intensa precipitación, de llovizna, de precipitación sólida y advecciones de aire frío durante el invierno, de tormentas de granizo, de advecciones de aire cálido y húmedo o aire frío y seco, de estabilidad invernal de tipo térmico, de subsidencias estivales de tipo dinámico y regímenes de brisas. Alusiones a algunas características peculiares de ciertos tipos de tiempo que pueden ser de gran ayuda en el estudio de situaciones sinópticas y en la determinación de la naturaleza de la masa de aire, hasta el punto de poder orientar hacia determinadas hipótesis en la investigación sinóptica del clima.

Por desgracia, un refrán no puede prestar más detalle que el de un par de premisas relacionadas vagamente entre sí, proceso incompleto si se compara con el minucioso examen científico de cualquier fenómeno climático, pero detrás se esconde la observación de generaciones durante siglos de experiencia, así el valor de la composición popular recae en el de una simple sentencia precedida de un profundo conocimiento del medio que todavía se conserva en ciertos ambientes rurales y marineros. Este artículo sólo se limita a ofrecer un ejemplo del análisis de las formaciones nubosas descritas en el refranero ${ }^{5}$, a costa de la dificultad que supone interpretar ciertas descripciones que resultan imprecisas y cuya relación con algunos tipos de tiempo es realmente ambigua. Pese a ello, las informaciones obtenidas en los refranes y adicionalmente en las encuestas del trabajo de campo, proporcionan elementos más que válidos para completar cualquier trabajo de Climatología, instrumentos que ningún científico puede eludir o desdeñar, más bien constituye una obligación el compromiso de su catalogación y estudio. Es necesario que los geógrafos, expertos conocedores de la relación que existe entre el medio físico y sus moradores, valoren esta fuente de información que es capaz de ofrecer vías de investigación que van más alla de los «fríos» tratamientos estadísticos de los datos de una garita. Después de trabajar durante dos años de esta manera, mirar al cielo a través de los ojos de los que sufrían directamente los efectos de la temperie obliga a verlo de una manera diferente y, con toda seguridad, más enriquecedora.

\section{Bibliografía}

CAPEL MOLINA, J. J.: «Insolación y nubosidad en la España peninsular y Baleares». Paralelo $37^{\circ}$ n. ${ }^{\circ}$ 1, pp. 9-24, Colegio Universitario de Almería, Departamento de Geografía 1977. CAPEL MOLINA J. J.: «Nubes cumuliformes». Paralelo $37^{\circ}$ n. ${ }^{\circ} 4$, pp. 5-18, Colegio Universitario de Almería, Departamento de Geografía, 1980.

\footnotetext{
${ }^{5}$ Los 1.700 refranes sobre el clima de la provincia de Alicante fueron la materia prima para el desarrollo de una memoria de licenciatura dirigida por el Dr. Gil Olcina y realizada durante un período que va desde enero de 1987 hasta marzo de 1989 en el Instituto Universitario de Geografía de la Universidad de Alicante. Actualmente se encuentra en prensa.
} 
CLAVERO PARICIO, P. L. y RASO NADAL, J. M.: «Catálogo de tipos sinópticos para un estudio climático del Este de la Península Ibérica y Baleares».Aportacions en homenatge al geògraf Salvador Llobet. Departament de Geografía. Ed. Universitat de Barcelona 1979.

Congreso sobre Avenidas fluviales e inundaciones en la cuenca del Mediterráneo. Instituto Universitario de Geografía de la Universidad de Alicante, Centre Européen de Coordination de Recherche et Documentation en Sciences Sociales (Viena) y la Caja de Ahorros del Mediterráneo, del 11 al 14 de mayo de 1988 en Alicante y Murcia.

GIL OLCINA, A.: «Lluvias excepcionales en la noche del 19 al 20 de octubre de 1982 y riada en el barranco de las Ovejas». Lluvias torrenciales e inundaciones en Alicante, Instituto Universitario de Geografía de la Universidad de Alicante, 1983.

GONZÁLEZ ARPIDE, J. L.: Los Tabarquinos (estudio etnológico de una comunidad en vías de desaparición.) Instituto de Estudios Alicantinos, Diputación Provincial de Alicante 1980.

GUILLÉN GARCÍA J.: El habla de Orihuela. Instituto de Estudios Alicantinos, Diputación Provincial de Alicante 1974.

I.N.M.: «Calendario meteorológico» años 1984-1987.

JANSA GUARDIOLA, J. M.: Manual del observador de Meteorología. Instituto Nacional de Meteorología, Madrid 1969 (2. edición).

LORENTE PÉREZ, J. M.: «Características meteorológicas en España de cada mes del año». Calendario Meteoro-fenológico 1944 a 1953, Servicio Meteorológico Nacional.

LLACER i BUENO, J. J.: Mil refranys de la Marina. Caja de Ahorros Provincial de Alicante, 1985.

MARTÍN VIDE, J.: Característiques climatològiques de la precipitació en la franja costera mediterrània de la Península Ibèrica. Institut Cartogràfic de Catalunya. Barcelona. 1987.

QUEREDA SALA, J.: «Los excepcionales temporales de octubre y su relación con las temperaturas del mar». Cuadernos de Geografía, n. ${ }^{\circ}$ 32-33, Valencia, 1983.

QUEREDA SALA, J.: El tiempo en la provincia de Castellón, Castellón, Exma. Dip. Prov. 1985.

SANCHIS GUARNER, M.: Calendari de refranys ed. Folklórica Barcino, Barcelona, 1951. TOHARIA, Manuel: Meteorología popular, ed. Observatorio, Madrid 1985.

TORREBLANCA ESPINOSA, M.: Estudio del habla de Villena y su comarca. Instituto de Estudios Alicantinos, Alicante 1976. 